\title{
Coinfección de tuberculosis y paratuberculosis en un toro de la Provincia del Chaco, Argentina
}

\author{
Martinez, D.E.; Cydezko, M.C.; Cipolini, M.F.; Jacobo, R.A.; Storani, C.A. \\ Cátedra de Enfermedades Infecciosas, Facultad de Ciencias Veterinarias, Universidad Nacional del Nordeste, \\ Cabral 2139, Corrientes (3400), Argentina. E-mail: dianaelina.dm@gmail.com
}

\begin{abstract}
Resumen
Martinez, D.E.; Cydezko, M.C.; Cipolini, M.F.; Jacobo, R.A.; Storani, C.A.: Coinfección de tuberculosis y paratuberculosis en un toro de la Provincia del Chaco, Argentina. Rev. vet. 27: 1, 48-50, 2016. Se describe la presentación de un caso de coinfección de tuberculosis y paratuberculosis en un toro Brangus de 8 años perteneciente a un rodeo de cría de Basail (Chaco, Argentina). El animal presentaba síntomas típicos de paratuberculosis - diarrea, emaciación, deshidratación crónica- y fue diagnosticado utilizando la intradermorreacción con PPD bovino para tuberculosis, ELISA indirecto en suero para paratuberculosis, así como necropsia e histopatología de órganos internos. El test ELISA resultó positivo y la tuberculinización negativa, sin embargo, el análisis histopatológico de muestras de pulmón e hígado permitió confirmar la presencia de lesiones compatibles con tuberculosis, mientras que las muestras de intestino y ganglios linfáticos mesentéricos revelaron evidencias de paratuberculosis.
\end{abstract}

Palabras clave: bovino, tuberculosis, paratuberculosis, serología, histopatología.

\begin{abstract}
Martinez, D.E.; Cydezko, M.C.; Cipolini, M.F., Jacobo, R.A.; Storani, C.A.: Coinfection of tuberculosis and paratuberculosis in a bull from Chaco Province, Argentina. Rev. vet. 27: 1, 48-50, 2016. A case of tuberculosis and paratuberculosis coinfection in a Brangus bull from Basail, Chaco (Argentina) is described. The animal presented typical symptoms of paratuberculosis - diarrhea, weight loss and dehydration - and was diagnosed by delayed hypersensitivity reaction with bovine PPD for tuberculosis, indirect ELISA for paratuberculosis, and necropsy and histopathology. Indirect ELISA resulted positive and delayed hypersensitivity reaction was negative. However, the histopathologic findings in lung and liver samples showed the presence of lesions compatible with tuberculosis, while intestine and mesenteric lymph nodes revealed evidences of paratuberculosis.
\end{abstract}

Key words: cattle, tuberculosis, paratuberculosis, serology, histopathology.

\section{INTRODUCCIÓN}

Tuberculosis (TBC) y paratuberculosis (PTBC) son enfermedades típicamente crónicas que afectan a los bovinos, a otras especies animales y al hombre. La TBC es una conocida zoonosis y la PTBC ha sido recientemente relacionada con la enfermedad de Crohn en seres humanos. Ambas son causadas por bacterias del género Mycobacterium: $M$. bovis en el caso de TBC y M. avium subespecie paratuberculosis en el de PTBC. En sendos casos se trata de bacilos ácido-alcohol resistentes (BAAR) que se caracterizan por poseer una gran cantidad de lípidos en su pared celular ${ }^{9}$.

Recibido: 22 diciembre 2015 / Aceptado: 2 marzo 2016
Si bien no existe una amplia información al respecto, la prevalencia de PTBC es del 7 al 19,6\% en rodeos de cría de Argentina ${ }^{5}$. En cuanto a TBC, se detectó un porcentaje promedio de bovinos reaccionantes a los diagnósticos de tuberculinización a campo de 1,6\% (rango: 1,5-1,7) en las provincias de Chaco y Formosa y de 6,1 (rango: 5,2-7,0) en la Provincia de Santa Fe ${ }^{10}$.

La TBC puede afectar varios órganos, pero generalmente compromete al pulmón, hígado y nódulos linfáticos, mientras que PTBC se circunscribe casi exclusivamente al intestino y sus ganglios linfáticos adyacentes. Ambas producen una reacción inflamatoria de tipo granulomatoso, aunque de características microscópicas diferentes: en TBC se define por focos de necrosis que pueden o no estar mineralizados, rodea- 
dos por células epitelioides, linfocitos y células gigantes de Langhans, cubiertos por una cápsula de tejido conectivo. Por su parte, PTBC causa un infiltrado de la mucosa del intestino, que puede ser focal o difuso y está compuesto por macrófagos, linfocitos y células gigantes de Langhans ${ }^{1,3}$.

\section{MATERIAL Y MÉTODOS}

Con motivo de un relevamiento sanitario se realizó la revisación clínica de un toro de raza Brangus perteneciente a un rodeo de cría de Basail (Provincia de Chaco, Argentina). El animal, de 8 años de edad y 590 $\mathrm{kg}$ de peso, presentaba a la inspección clínica facie apagada, marcha lenta y dificultosa, así como un crítico estado de nutrición caracterizado por intenso adelgazamiento. El pelaje estaba opaco, ralo e hirsuto.

Al momento de la inspección se observó una deyección líquida, verdosa y maloliente, que caracterizaba el cuadro clínico. Las mucosas aparentes se encontraban pálidas, existía una deshidratación moderada y la temperatura corporal se situó dentro del rango fisiológico $\left(39^{\circ} \mathrm{C}\right)$. Su apetito era normal.

Ante la presunción de que el cuadro clínico correspondiera a PTBC, y a los efectos de confirmar el diagnóstico, se extrajo una muestra de sangre cuyo suero fue procesado según test de ELISA indirecto (enzymelinked immuno sorbent assay) utilizando un kit comercial (ID VET $\left.{ }^{\circledR}\right)$. Dado que la TBC se incluye en el diagnóstico diferencial, se realizó la tuberculinización del animal utilizando $0,1 \mathrm{ml}$ de PPD bovino (purified protein derivative, Tuberculina Test ${ }^{\circledR}$, Merial), aplicado en el pliegue ano-caudal interno, efectuándose la lectura a las $72 \mathrm{~h}$ según reglamentación ${ }^{8}$.

Con posterioridad se realizó la necropsia del animal enfermo, la identificación de lesiones macroscópicas y la extracción de muestras para análisis anatomopatológico.

\section{RESULTADOS}

La tuberculinización arrojó resultado negativo pero el test ELISA fue positivo. En la necropsia, la apertura de la cavidad abdominal permitió visualizar tejido adiposo de color amarillo muy intenso, congruente con el adelgazamiento progresivo del animal. Los intestinos presentaban ondulaciones anómalas en su superficie. Al corte, la mucosa del intestino presentaba las lesiones "cerebriformes" (Figura 1) características de la PTBC, alteraciones que se observaron a lo largo de todo el órgano.

Los ganglios linfáticos del mesenterio ostentaban una coloración pardo-negruzca. El hígado, en la superficie diafragmática del lóbulo izquierdo, presentaba lesiones circulares de $5 \mathrm{~cm}$ de diámetro y color blanquecino, visibles a través de la cápsula. Además exhibía lesiones puntiformes de $0,5 \mathrm{~cm}$ de diámetro de color blanquecino en el parénquima.
En el pulmón izquierdo se hallaron múltiples áreas hepatizadas de color rosado, de $10 \mathrm{~cm}$ de diámetro aproximadamente, que al corte evidenciaron varios nódulos blanquecinos, esféricos, de $1 \mathrm{~cm}$ de diámetro, con un contenido de aspecto caseoso (Figura 2). Los demás órganos no presentaron alteraciones visibles.

En todas las muestras de intestino delgado (principalmente íleon) y grueso, los preparados histológicos revelaron una arquitectura parcialmente alterada por la presencia de abundantes macrófagos y linfocitos en la lámina propia de la mucosa y en la submucosa. Las secciones coloreadas con Ziehl Neelsen revelaron la presencia de BAAR en el citoplasma de los macrófagos.

En las muestras de nódulos linfáticos se detectó la presencia de macrófagos en los senos con BAAR. El hígado presentó un foco con material necrótico rodeado de infiltrado inflamatorio monomorfonuclear y abundante tejido conectivo. Además, en los espacios porta se observaron pequeños focos de infiltrado monomorfonuclear.

El pulmón mostró una arquitectura alterada por la presencia de granulomas con células gigantes multinucleadas de tipo Langhans, con áreas de calcificación.

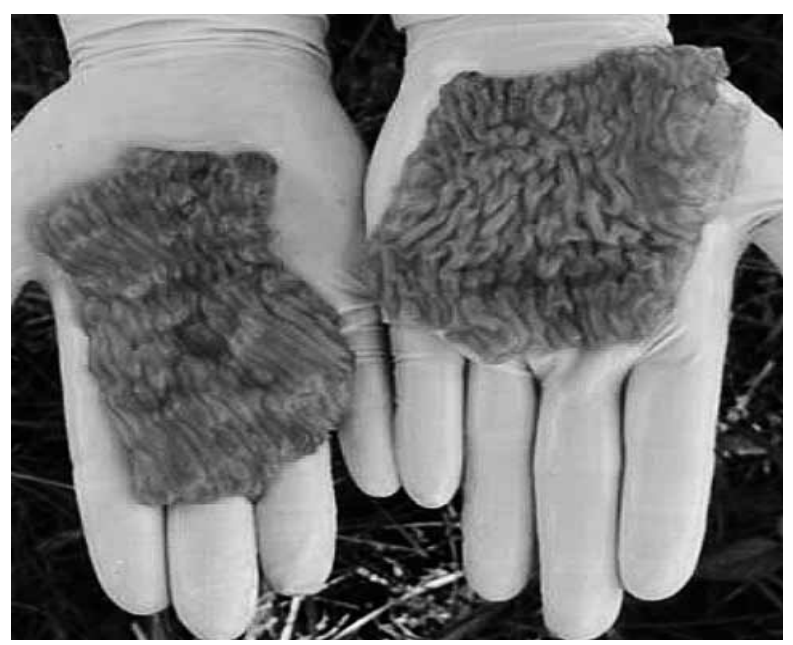

Figura 1. Lesiones "cerebriformes" en intestino delgado, características de PTB.

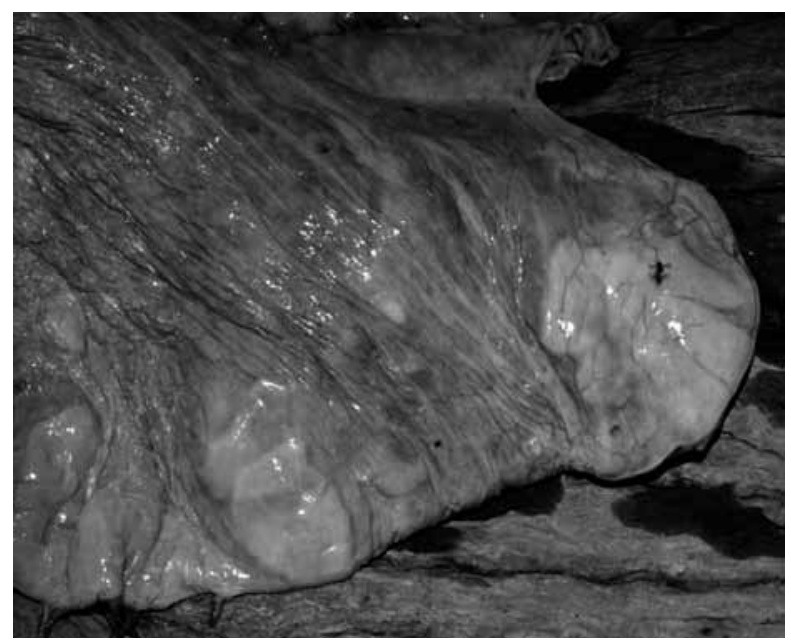

Figura 2. Lesiones granulomatosas en pulmón, compatibles con TBC. 
Se observaron focos de material necrótico en la pared y luz de los bronquiolos. No se detectaron BAAR. Las lesiones se identificaron como enteritis y linfoadenitis crónicas granulomatosas con presencia de BAAR, vinculables con PTBC, y a su vez neumonía, así como hepatitis crónica granulomatosa, compatibles con TBC.

Los hallazgos serológicos y las lesiones histopatológicas permitieron diagnosticar una coinfección de tuberculosis y paratuberculosis en el toro.

\section{DISCUSIÓN}

La negatividad al test tuberculínico en el animal enfermo de TBC, podría deberse al fenómeno de anergia que caracteriza a los estados crónicos de la enfermedad y obedece a un detrimento importante en la capacidad de respuesta del sistema inmune ${ }^{6}$. La coinfección con M. avium supesp. paratuberculosis podría haber contribuido a esta imposibilidad de reaccionar ante el test tuberculínico.

Es sabido que en los estados subclínicos de la PTBC se activa una respuesta celular mientras que en la fase clínica, cuando se presenta la diarrea y la pérdida de peso, la respuesta celular declina para dar paso a una manifestación de inmunidad humoral, con aumento de anticuerpos específicos que pueden ser detectados mediante el test de ELISA ${ }^{2}$. El test de ELISA I detecta animales clínicamente enfermos o subclínicamente infectados, con una sensibilidad de $66 \%$ y especificidad de $99 \%{ }^{4}$.

En conclusión, este caso alerta sobre la necesidad de establecer un sistema de control de ambas enfermedades, que podría combinar varios métodos de diagnóstico, incluyendo la tuberculinización, la serología y los cultivos fecales ${ }^{7}$, a efectos de aumentar las chances de identificación de animales positivos en etapas tempranas y así evitar la diseminación ambiental de ambas enfermedades.

\section{REFERENCIAS}

1. Chiodini RJ, van Kruiningen HJ, Merkal R. 1984. Ruminant paratuberculosis (Johne's disease): the current status and future prospects. Cornell Vet 74: 218-262.
2. Cocito C, Gilot P, Coene M, Kesel M, Poupart P, Vannuffel P. 1994: Paratuberculosis. Clin Microbiol Rev 7: 328-345.

3. Dungworth DL. 1985. The respiratory system. In: Pathology of Domestic Animals (Jubb K, Kennedy P, Palmer N, Ed), 3rd. ed., Vol. 2, Acad. Press, Orlando (USA), p. 493505.

4. Martinis Mercado DS, Cicuta ME, Boehringer SI, Morsella C, Paolicchi F. 2002. La paratuberculosis y los bovinos lecheros de la Provincia de Corrientes. Anales Sesión Comunic. Cientif. Fac.Cs.Vet.UNNE (Corrientes, Argentina), p. 52.

5. Moreira A, Spath E, Morsella C. 1994. Seroprevalence of Johne's disease in eleven districts of Buenos Aires, Argentina. In: Proceedings of the Fourth International Colloquium on Paratuberculosis (Chiodini R, Collins M, Bassey E. eds), p. 33, Cambridge, UK.

6. O'Reilly L, Daborn M. 1995. The epidemiology of Mycobacterium bovis infections in animals and man: a review. Tubercle and Lung Disease: 76 (Supplement 1): 1-46.

7. Paolicchi F, Zumarraga A, Gioffre MJ, Zamorano A, Morsella P, Verna C, Cataldi A, Alito A, Romano M. 2003. Application of different methods for the diagnosis of paratuberculosis in a dairy cattle herd in Argentina. $J$ Vet Med B 50: 20-26.

8. Servicio Nacional de Sanidad y Calidad Agroalimentaria (SENASA, Argentina). 2012. Plan nacional de control y erradicación de la tuberculosis bovina. Resolución 128/12.

9. Thorel M, Krichevsky M, Lévy-Frébault V. 1990. Numerical taxonomy of micobactin-dependent mycobacteria, emended description of Mycobacterium avium, and description of Mycobacterium avium subsp. avium subsp. nov., Mycobacterium avium subsp. paratuberculosis subsp. nov. and Mycobacterium avium subsp. silvaticum subsp. nov. Int J Sys Bacteriol 40: 254-260.

10. Torres PM. 2014. Situación de la tuberculosis bovina en la República Argentina. Presentado en el $I I I^{\circ}$ Congreso Panamericano de Zoonosis (4-6/06/2014, La Plata, Argentina), Anales, 31 p. On line: ps://viejaweb.senasa.gov. ar/Archivos/File/File 1013-situacion_tuberculosis_bovina_junio_2011.pdf 